\title{
Contrast-enhanced MRI of the knee in children unaffected by clinical arthritis compared to clinically active juvenile idiopathic arthritis patients
}

\author{
Charlotte M. Nusman ${ }^{1,2} \cdot$ Robert Hemke $^{1,2}$ - Marc A. Benninga ${ }^{3}$. \\ Dieneke Schonenberg-Meinema $^{2}$ - Angelika Kindermann ${ }^{3}$ • Marion A. J. van Rossum ${ }^{2,4}$. \\ J. Merlijn van den Berg ${ }^{2} \cdot$ Mario Maas $^{1}$ - Taco W. Kuijpers ${ }^{2}$
}

Received: 9 January 2015 /Revised: 26 June 2015 / Accepted: 30 June 2015 /Published online: 2 August 2015

(C) The Author(s) 2015. This article is published with open access at Springerlink.com

\begin{abstract}
Objectives To evaluate enhancing synovial thickness upon contrast-enhanced magnetic resonance imaging (MRI) of the knee in children unaffected by clinical arthritis compared with clinically active juvenile idiopathic arthritis (JIA) patients. A secondary objective was optimization of the scoring method based on maximizing differences on MRI between these groups.

Methods Twenty-five children without history of joint complaints nor any clinical signs of joint inflammation were age/ sex-matched with 25 clinically active JIA patients with arthritis of at least one knee. Two trained radiologists, blinded for clinical status, independently evaluated location and extent of enhancing synovial thickness with the validated Juvenile Arthritis MRI Scoring system (JAMRIS) on contrastenhanced axial fat-saturated T1-weighted MRI of the knee.
\end{abstract}

Mario Maas and Taco W. Kuijpers contributed equally to this work.

Electronic supplementary material The online version of this article (doi:10.1007/s00330-015-3912-z) contains supplementary material, which is available to authorized users.

Charlotte M. Nusman

c.m.nusman@amc.nl

1 Department of Radiology, Academic Medical Center, University of Amsterdam, Meibergdreef 9, 1105 AZ Amsterdam, The Netherlands

2 Department of Pediatric Hematology, Immunology, Rheumatology and Infectious Disease, Emma Children's Hospital AMC, University of Amsterdam, Meibergdreef 9, Amsterdam, The Netherlands

3 Department of Pediatric Gastroenterology, Emma Children's Hospital AMC, University of Amsterdam, Meibergdreef 9, Amsterdam, The Netherlands

4 Department of Pediatric Rheumatology, Reade, Dr. Jan van Breemenstraat 2, Amsterdam, The Netherlands
Results Enhancing synovium $(\geq 2 \mathrm{~mm})$ was present in 13 (52\%) unaffected children. Using the total JAMRIS score for synovial thickening, no significant difference was found between unaffected children and active JIA patients ( $p=$ 0.091). Additional weighting of synovial thickening at the JIA-specific locations enabled more sensitive discrimination $(p=0.011)$.

Conclusions Mild synovial thickening is commonly present in the knee of children unaffected by clinical arthritis. The infrapatellar and cruciate ligament synovial involvement were specific for JIA, which — in a revised JAMRIS - increases the ability to discriminate between JIA and unaffected children.

Key Points

- Synovial inflammation is the primary disease feature in juvenile idiopathic arthritis (JIA).

- Appearance of the synovium on contrast-enhanced MRI in unaffected children is unknown.

- Validation of existing scoring methods requires comparison between JIA and unaffected children.

- Mild enhancing synovial thickening was detected in half of the unaffected children.

- Location-weighting for JIA-specific locations increased discriminative value of the scoring methods $(p=0.011)$.

Keywords Magnetic resonance imaging · Juvenile idiopathic arthritis $\cdot$ Knee $\cdot$ Control group $\cdot$ Synovium
Abbreviations
IBD
IV
Inflammatory bowel disease
JADAS Intravenous
JAMRIS Juvenile Arthritis Disease Activity Score
JIA Juvenile Arthritis MRI Scoring system Juvenile idiopathic arthritis 


$\begin{array}{ll}\text { MRE } & \begin{array}{l}\text { Magnetic resonance enterography } \\ \text { MRI }\end{array} \\ \text { OMERACT } & \begin{array}{l}\text { Oagnetic resonance imaging } \\ \text { Trials }\end{array} \\ \text { PCDAI } & \text { Paediatric Crohn's Disease Activity Index } \\ \text { PUCAI } & \text { Paediatric Ulcerative Colitis Activity Index } \\ \text { pGALS } & \text { Paediatric Gait Arms Legs Spine } \\ \text { SD } & \text { Standard deviations } \\ \text { TE } & \text { Echo time } \\ \text { TR } & \text { Repetition time }\end{array}$

\section{Introduction}

Juvenile idiopathic arthritis (JIA) is the most common form of childhood arthritis and is characterized by inflammation of the synovium. Early and aggressive treatment is warranted for the prevention of permanent joint damage and the achievement of inactive disease [1-3]. Magnetic resonance imaging (MRI) is currently state of the art in JIA, especially in early stage disease [4]. Additionally, MRI scans of the knee of JIA patients are easily, reliably and quantitatively assessed by means of the Juvenile Arthritis MRI Scoring system (JAMRIS), which is a composite score on four domains (synovial thickening upon contrast enhancement, bone marrow oedema, cartilage lesions and bone erosions) [5]. Although the intravenous (IV) administration of contrast is a disadvantage of MRI in the assessment of disease activity in JIA patients, it is essential for adequate depiction of the synovium as the primary target of disease [6-8].

After selecting relevant features and reliability studies of the current scoring method JAMRIS, the next step in the validation is the comparison of JIA and children unaffected by clinical arthritis [5, 9]. The need for data regarding the appearance of healthy paediatric joints upon MRI was also emphasized by the Outcome Measures in Rheumatology Clinical Trials (OMERACT) Special Interest Group for MRI in JIA [10], especially because enhancing synovial thickening on MRI after IV contrast administration was commonly observed in previous MRI studies in healthy adults [11-13].

Awareness of the appearance of the enhancing synovium in unaffected children is crucial to differentiate between physiologic and pathologic thickness of the synovium. An accurate distinction between these two may have important implications for treatment decisions in JIA patients. Currently, the differentiation between normal synovium and synovial thickening with JAMRIS is made on the basis of a cut-off value for thickness of pathologically enhancing synovium of $2.0 \mathrm{~mm}$, as assessed in JIA patients only [5]. However, this cut-off value is arbitrary as knowledge of the synovial thickness on MRI in healthy children is lacking. Performing contrastenhanced MRI of the knee in healthy children was considered unethical by our institutional review board. To assess the appearance of the synovium by MRI in children unaffected by clinical arthritis, we included patients suspected or diagnosed with inflammatory bowel disease (IBD) who underwent magnetic resonance enterography (MRE) with IV contrast.

Our study design obviously needed precautionary measures to ensure that the appearance of the synovium mimics that of the healthy population as well as possible, e.g. with respect to timing of IV contrast and exclusion of joint complaints. The objectives of this study were (1) to evaluate enhancing synovial thickness upon contrast-enhanced MRI of the knee in children unaffected by clinical arthritis compared with clinically active JIA patients and (2) to explore the possibilities to optimize JAMRIS by maximizing the difference in synovial thickening between unaffected children and clinically active JIA patients.

\section{Materials and methods}

\section{Children unaffected by clinical arthritis}

The cohort of children unaffected by clinical arthritis consisted of prospectively included children suspected or diagnosed with IBD between 8 and 18 years of age, who underwent MRE with IV contrast between April 2012 and March 2014. The activity of the underlying IBD was evaluated with either the Paediatric Crohn's Disease Activity Index (PCDAI) or the Paediatric Ulcerative Colitis Activity Index (PUCAI) [14, 15]. The PCDAI ranges from 0 to 100 points (with a score of less than 10 considered inactive disease, 1030 mild disease and above 30 moderate to severe disease) and the PUCAI ranges from 0 to 85 points (similar disease activity cut-off values). Exclusion criteria for this cohort of children unaffected by clinical arthritis were congenital or genetic joint abnormalities, a history of trauma in both knees (if only one knee suffered trauma, the other knee was imaged), and any arthritic diseases or other chronic conditions affecting the skeleton. The physical examination was performed by a research fellow trained by an experienced paediatric rheumatologist (25 years of experience) and by means of the validated paediatric Gait Arms Legs Spine (pGALS) screening tool [16]. Both exclusion criteria and physical examination were used to ensure that the joints of these patients undergoing MRE were asymptomatic. Medication use and indication for MRI were registered as well.

The MRI examinations were performed on a 1.5-T MRI scanner (MAGNETOM Avanto ${ }^{\mathrm{TM}}$ 1.5 T MRI, Siemens Medical Systems). None of the patients received anaesthetics for their MRI. After the regular MRE with IV contrast injection (Gadovist, Bayer Schering Pharma, Berlin, Germany, $1.0 \mathrm{mmol}$ gadolinium $/ \mathrm{mL}$, dose $0.1 \mathrm{mmol} / \mathrm{kg}$ of body weight), a dedicated knee coil was connected. The MRE sequences after injection of IV contrast were a coronal and axial T1 
VIBE sequence with fat saturation (approximately $4 \mathrm{~min}$ together). An axial T1-weighted sequence with fat saturation of the right knee was performed (TR 400-750 ms, TE $10 \mathrm{~ms}$; slice thickness $4 \mathrm{~mm}$; field of view $150 \times 150 \mathrm{~mm}$, matrix $384 \times 384)$. If the patient suffered recent trauma in the right knee (and therefore disturbing the healthy appearance), the left knee was imaged. Time between injection of IV contrast and start of the MRI sequence of the knee was registered. This study was approved by the institutional review board and all participants (and their parents if the patients were younger than 16 years old) gave written informed consent.

\section{Clinically active JIA patients}

JIA patients with clinical arthritis of at least one knee (henceforth referred to as 'clinically active JIA patients') were derived from our MRI in JIA study database. Patients who consecutively visited from April 2012 (start inclusion asymptomatic controls) and who underwent an MRI scan of the knee were age- and sex-matched to the asymptomatic control cohort. Clinical characteristics have been prospectively collected in our MRI in JIA study database. Reported in this study are the JIA subtype, disease duration (from disease onset until MRI), medication use and Juvenile Arthritis Disease Activity Score (JADAS). The JADAS-10 (ranging from 0 to 40 ) is the sum of the scores of the physician and parent/patient global assessment of disease activity, reduced 10-active joint count and a normalized value of erythrocyte sedimentation rate to a $0-10$ scale [17]. All parents of the JIA patients and JIA patients older than 12 years gave written informed consent.

The imaging protocol of the MRI of the knee in JIA patients on the open-bore 1.0-T scanner (Panorama HFO, Philips Healthcare) consists of six sequences. For comparison with the unaffected children, only the axial T1-weighted sequence with fat saturation after IV contrast was used (Gadovist, Bayer
Schering Pharma, Berlin, Germany, $1.0 \mathrm{mmol}$ gadolinium/ $\mathrm{mL}$, dose $0.1 \mathrm{mmol} / \mathrm{kg}$ ). Parameters of this sequence (TR, TE, slice thickness, field of view) were similar to the sequence performed in the unaffected children.

\section{Image analysis}

The synovium on MRI of the knee was evaluated on six welldefined locations (suprapatellar, patellofemoral, infrapatellar, cruciate ligaments, medial posterior condyle and lateral posterior condyle) following the validated JAMRIS system for the knee (Fig. 1) [5]. The thickness of the enhancing synovium was, if possible, measured at each location and scored following the JAMRIS system ( 0 , enhancing synovium less than $2 \mathrm{~mm}$; 1, enhancing synovium from at least 2 to $4 \mathrm{~mm} ; 2$, enhancing synovium at least $4 \mathrm{~mm}$ ) [5]. The total JAMRIS score consists of the sum of the scores for enhancing synovium at all six locations.

The MRI data sets of the knee of both the unaffected children and the JIA patients were blinded (for age, gender and asymptomatic/JIA group) and randomized. Two experienced readers (radiologists with 6 and 19 years of experience in musculoskeletal radiology) independently evaluated all images. Agreement on the incongruent cases (difference between readers in total JAMRIS score greater than 1) was obtained afterwards in a consensus reading. If the difference in total JAMRIS score between the readers was less than 1, the score of the most experienced reader was adopted.

Possibilities to optimize the JAMRIS score were explored by means of a concept which was inspired by previous work on preferred locations for JIA on MRI [18]. The preferred locations were identified on the basis of a significantly higher JAMRIS score in the JIA patients compared to the unaffected children. For these locations we applied a weighting measure; this simple JAMRIS revision comprised the doubling of the score for enhancing synovial thickening at the JIA-specific

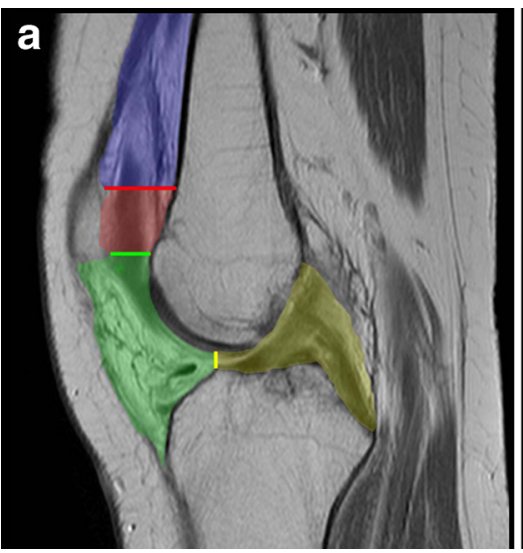

Fig. 1 The locations at which enhancing synovial thickening on MRI of the knee is scored according to the Juvenile Arthritis MRI Scoring system (JAMRIS) [5]. On a sagittal T1-weighted sequence (a) and an axial fatsaturated T1-weighted sequence (b) of the knee the six locations are

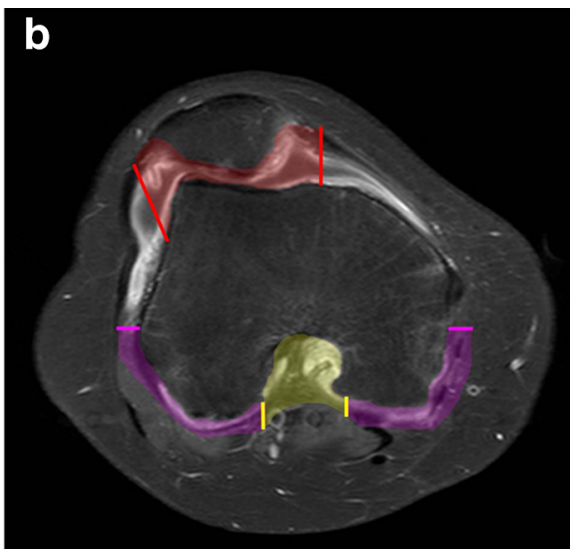

exemplified with different colours: blue suprapatellar, red patellofemoral, green infrapatellar, yellow cruciate ligaments, purple medial and lateral posterior condyle 
infrapatellar and/or cruciate ligament locations, meaning that an additional point was added to the total score when contrastenhanced thickening of the synovium (at least $2 \mathrm{~mm}$ ) was only present at these two JIA-specific locations [18].

\section{Statistical analysis}

Descriptive statistics (mean, standard deviation (SD), percentages) were used to describe baseline characteristics and MRI findings. Bland-Altman plots were used to assess the agreement between the two readers. Mann-Whitney $U$ test was used to analyse differences between the groups in total MRI scores and time between injection of contrast and start of MRI sequence. The Pearson correlation coefficient was used to assess the correlation between mean synovial thickness and the time between injection of contrast and start of MRI sequence, because the timing can influence the appearance of the synovium [19]. All data were analysed using SPSS software, version 20 (SPSS, Chicago, IL, USA).

\section{Results}

\section{Patient characteristics}

Twenty-five children unaffected by clinical arthritis previously diagnosed with ( $68 \%$ ) or suspected of having (32\%) IBD underwent MRE (for diagnosis or evaluation of the extent of the IBD), followed by an additional MRI sequence of the knee. Clinical arthritis in these children unaffected by JIA was ruled out through stringent exclusion criteria, questionnaires and simultaneous physical examination at the moment of imaging. For comparison, 25 age- and sex-matched clinically active JIA patients were included in the study. Baseline characteristics of both groups are summarized in Table 1.

\section{Overall image analysis}

The total JAMRIS scores for synovial thickening of the two readers corresponded (difference in total score at most 1 ) in 38 of the 50 MRI data sets. In 25/38 data sets the difference in total JAMRIS score was 1 , so the score of the most senior reader was adopted. The agreement between the two readers was very good, as depicted in the Bland-Altman plot in Supplementary File 1.

\section{Imaging findings}

Enhanced thickening (at least $2 \mathrm{~mm}$ ) of the synovium on at least one out of six well-defined locations was present upon MR imaging of the knee in 13/25 (52\%) of the unaffected children (Fig. 2), compared to 16/25 (64\%) of the active JIA patients. The maximum total JAMRIS score for synovial
Table 1 Baseline characteristics of children unaffected by clinical arthritis and clinically active JIA patients

\begin{tabular}{lll}
\hline & $\begin{array}{l}\text { Unaffected } \\
\text { children }\end{array}$ & $\begin{array}{l}\text { Clinically active } \\
\text { JIA patients }\end{array}$ \\
\hline Subjects & 25 & 25 \\
Female, $n(\%)$ & $11(44)$ & $11(44)$ \\
Age at MRI, years & $13.5( \pm 2.5)$ & $13.3( \pm 2.5)$ \\
IV contrast - MRI knee, minutes & $10.5( \pm 2.4)$ & $4.9( \pm 1.1)^{*}$ \\
Immunomodulatory drugs ${ }^{\mathrm{a}}, n(\%)$ & $15(60)$ & $8(32)$ \\
PCDAI $(n=19)$ & $23( \pm 14)$ & - \\
PUCAI $(n=6)$ & $29( \pm 28)$ & - \\
Diagnosis IBD ${ }^{\mathrm{b}}, n(\%)$ & & - \\
Crohn's disease & $18(72.0)$ & - \\
Ulcerative colitis & $4(16.0)$ & - \\
No or other disorder & $3(12.0)$ & - \\
JIA disease duration, months & - & $11( \pm 6)$ \\
JADAS-10 $(0-40)$ & - & $4(16)$ \\
Diagnosis JIA, $n(\%)$ & & $1(4)$ \\
Oligo-articular persistent & - & $1(40)$ \\
Oligo-articular extended & - & \\
Poly-articular RF- & - & \\
Poly-articular RF+ & - & \\
ERA & - & \\
Unclassified & - & \\
\hline
\end{tabular}

Unless indicated otherwise, values are mean (SD)

$E R A$ enthesitis-related arthritis, $I B D$ inflammatory bowel disease, $I V$ intravenous, JADAS Juvenile Arthritis Disease Activity Score, JIA juvenile idiopathic arthritis, PCDAI Paediatric Crohn's Disease Activity Index, $P U C A I$ Paediatric Ulcerative Colitis Activity Index, $R F$ rheumatoid factor

${ }^{*} p<0.05$

${ }^{\text {a }}$ Immunomodulatory drugs in the children unaffected by JIA included mesalazine (in 4 children), azathioprine (in 7 children, dosed at 2.0 $2.5 \mathrm{mg} / \mathrm{kg}$ ) and prednisone (in 4 children at $0.8,1.0$ and $5.0 \mathrm{mg} / \mathrm{kg}$, respectively) for IBD, whereas in JIA the drugs methothrexate (at 10 $15 \mathrm{mg} / \mathrm{m}^{2}$ per week, in 7 patients) and/or anti-tumour necrosis factor (TNF)-alpha (in 2 patients) were used

b These numbers represent the final diagnosis, not the diagnosis at time of MRE

thickening in unaffected children was 3 , with no location scoring higher than 1 (i.e. less than $4 \mathrm{~mm}$ ).

Upon analysis of the total population (including both unaffected children and JIA patients), we found no correlation between the time (in minutes) between injection of IV contrast and start of the MRI knee sequence and the mean synovial thickness per reader (correlation coefficients -0.013 and $-0.140, p=0.378-0.929)$. Furthermore, when only evaluating the unaffected children and dividing them into those requiring less than $10 \mathrm{~min}$ and those requiring at least $10 \mathrm{~min}$ between injection of contrast and start of the MRI sequence, no significant differences were found in the total JAMRIS score for 


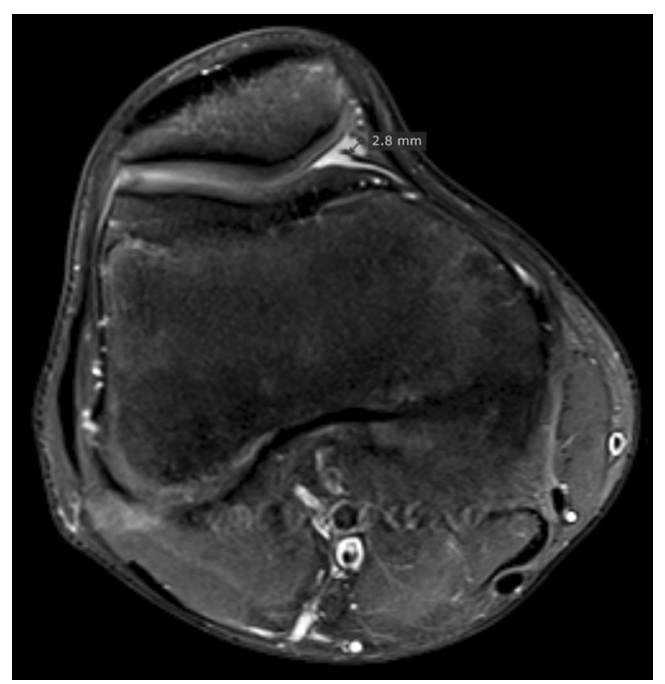

Fig. 2 A 12-year-old boy unaffected by JIA shows presence of enhancing synovial thickening greater than $2 \mathrm{~mm}$ on an axial T1-weighted MR image of the knee

synovitis $(p=0.403$ ) or the mean synovial thickness per reader $(p=0.126$ and $p=0.341)$.

Also the use of immunomodulatory drugs (i.e. azathioprine and prednisone) in the unaffected children did not result in a suppression of the contrast-enhancement of the synovium in the knee, because no difference in total JAMRIS score of was found between unaffected children with and those without taking immunomodulatory drugs $(p=0.120)$.

Being confident about the data quality and ability to compare the imaging data obtained from the different cohorts, we analysed these data in greater detail. We observed a surprisingly large overlap of the total JAMRIS score between active JIA patients and children unaffected by clinical arthritis (Fig. 3), which was reflected by a lack of a significant difference in the absolute total JAMRIS score as currently used for synovial thickening ( $p=0.091)$. Thirty-six per cent of the active JIA patients showed no synovial thickening on MRI (total JAMRIS score 0).

Although the total JAMRIS scores for synovial thickening were within the same range, when unaffected children and active JIA patients were analysed per location, the mean JAMRIS score was significantly different at two out of six locations: infrapatellar $(p=0.011)$ and cruciate ligaments $(p=$ 0.007). An example of this typical enhancing thickened synovium at the infrapatellar and cruciate ligament location in a JIA patient is given in Fig. 4. To optimize the JAMRIS score by maximizing the differences between active JIA and unaffected children, we applied a weighting measure for the locations with enhancing thickened synovium in JIA cases only. This revised JAMRIS score with a doubling of the score for infrapatellar and cruciate ligament locations resulted in considerably less overlap between active JIA and unaffected children (Fig. 5) with a significant difference between groups $(p=0.011)$.

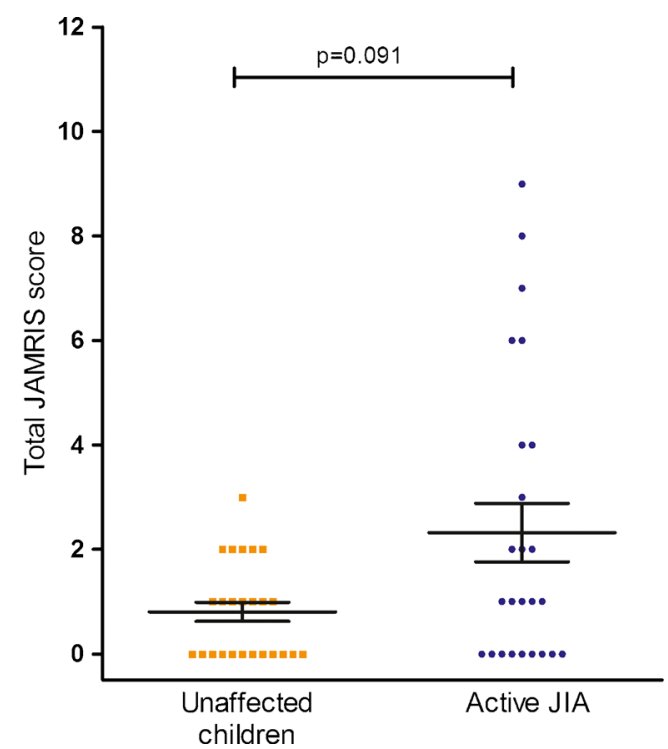

Fig. 3 The total JAMRIS scores for synovial thickening with mean and standard error of the mean (represented by the black lines) in the unaffected children (orange) and active JIA patients (blue)

The infrapatellar region was highly specific for JIA as it was never affected in any of the children without clinical arthritis. Increased signal enhancement at this infrapatellar region was not mistaken for fat pad oedema due to patellar maltracking, because this oedema occurs in the superolateral aspect of the fat pad and the synovial thickening we scored only occurred in the central part of the infrapatellar region [20].

\section{Discussion}

This study is the first to determine the appearance of the synovium on contrast-enhanced MRI comparing clinically active JIA patients with children unaffected by clinical arthritis. Enhancing synovium (at least $2 \mathrm{~mm}$ ) was found to be present in the majority of unaffected children. Although the total score for synovial thickening showed a major overlap between active JIA patients and unaffected children, there were noticeable JIA-specific properties of JAMRIS, including a total score of greater than 3, a score of 2 at any of the six locations and isolated presence of enhancing thickened synovium at the infrapatellar region and/or near the cruciate ligaments. A simple revision of JAMRIS using a certain weighting of these unique and JIA-specific locations resulted in an improvement in the possibility to discriminate between active JIA patients and unaffected children.

To ensure that the presence of enhancing synovium in our study population is representative for all children unaffected by JIA, factors possibly contributing to a high incidence of synovial contrast enhancement were ruled out. The most important factor with a possible influence on the thickness of 


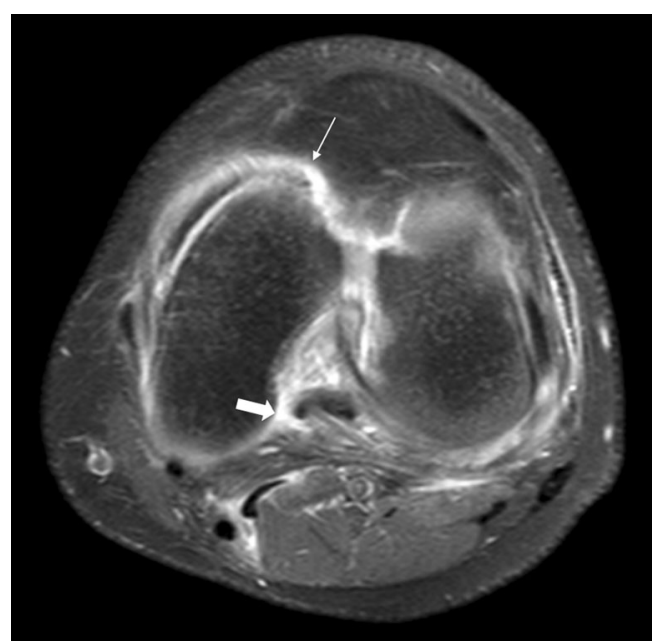

Fig. 4 An example of the axial T1-weighted MRI sequence of the knee in a 15-year-old clinically active JIA patient with typical enhancing and thickened synovium at the cruciate ligament location (thick arrow) and the infrapatellar location (thin arrow)

enhancing synovium on MRI was considered to be the timing of the administration of IV contrast [19]. As a result of the necessity to change the body coil after MRE to a dedicated knee coil, timing was significantly different between the two groups. This leads to a possible 'overestimation' of the synovial thickness in the unaffected children, as the contrast diffuses out of the synovial membrane over time. There is no consensus on the timing of IV contrast in the literature, but it is said that the distinction between synovium and effusion can be reliably made within the first 15 min after administration of IV contrast [19, 21]. While there was no association between

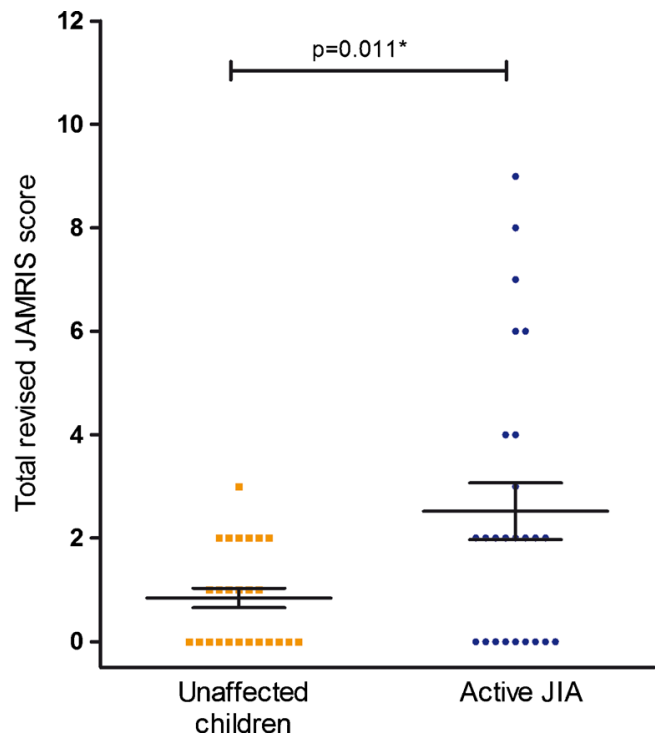

Fig. 5 The total revised JAMRIS scores for synovial thickening with mean and standard error of the mean (represented by the black lines) in the unaffected children (orange) and active JIA patients (blue). The revised JAMRIS score comprised a weighting of the isolated presence of synovial thickening at the infrapatellar and/or cruciate ligament location the time between injection of IV contrast and the MRI sequence (in minutes) and the mean synovial thickness per reader or total JAMRIS score, this factor was believed not to influence the interpretation of synovial thickness in our study population.

The other factor possibly contributing to the high incidence of enhancing thickened synovium was the patient population itself. The necessity of an IV contrast agent for adequate visualization of the synovium on MRI creates a difficult ethical issue, as the administration of IV contrast and the invasiveness of an infusion are not desirable in completely healthy children without any disease manifestation. For the design of this imaging study, we hence selected a population of children without joint complaints, with a relatively low disease burden and already receiving an MRI with IV contrast for regular patient care. The risk of signs of arthritis on MRI in our paediatric control group was maximally reduced, having excluded patients with any joint complaints at any time. These unaffected were children suspected of having or diagnosed with IBD, who may hence suffer from an autoimmune inflammatory disease burden similar to patients with JIA. However, strict exclusion criteria and thorough physical examination by means of a validated checklist (pGALS) were undertaken to avoid any chance of clinical arthritis in these children unaffected by JIA [16]. In large registry studies, the incidence of clinical arthritis among IBD patients has repeatedly been reported to be very low (less than $4 \%$ ) [22, 23].

Considering the aforementioned methodological issues of little if any relevance for the data analysis, the outcome of the results needs further discussion. The presence of enhancing synovial thickening in children unaffected by clinical arthritis was reminiscent of previous studies in adults that suggested synovial thickening was present upon MRI of the knee in adult healthy controls $[13,24]$. The finding of minor synovial thickening (total score less than 3 ) in the unaffected children would not cause any problem if the total score with JAMRIS for synovial thickening in the active JIA patients were significantly higher. However, the results of our study showed large overlap in scores between these children unaffected by clinical arthritis and active JIA patients. This could be explained in several ways.

First, synovial thickening on MRI may not be present in all clinically active JIA patients. Our results showed that in $32 \%$ of the clinically active JIA patients synovial thickening upon MRI of the knee was found to be absent (JAMRIS score 0). This discrepancy between clinical findings and imaging results was reported in several previous studies as well [18, $25,26]$. Ethical issues will prevent us from comparing biopsy-proven synovial changes in JIA versus control tissue samples. The lack of this true gold standard for the presence of synovitis in the paediatric joint would thus impede full clarification of the absence of synovial thickening on MRI in clinically active JIA patients and justifies our current studies in 
unaffected children. Additional ongoing follow-up studies in larger JIA study samples are needed to clarify whether these MRI-negative yet clinically active JIA patients are more likely to represent a subgroup with a different type of joint pathology.

Secondly, the overlap between the appearance of the synovium upon imaging of unaffected children and active JIA patients could be explained by too low a cut-off value for pathologic synovial thickness $(2.0 \mathrm{~mm}$, as previously proposed in JAMRIS) [5]. However, on the basis of the absolute measures of synovial thickness per location for both readers, no other (higher) appropriate cut-off value would lead to a more distinct difference between unaffected children and active JIA (data not shown).

To maximize the difference between a healthy condition and pathology by means of the JAMRIS scoring method, we decided to give more weight to the JIA-specific locations of synovial thickening that we identified in the current study which were completely absent in any of the controls. Our study on the typical distribution of MRI abnormalities in JIA already indicated a noticeable preference for synovial thickening at the central locations in the knee [18]. Applying a simple location-weighting as a simple revision of JAMRIS by adding a point to the total score if synovial thickening was solely present per infrapatellar and/or cruciate ligaments location already proved to differentiate the unaffected children from the active JIA patients. This now legitimizes further exploration of this location-weighting approach in larger JIA study samples (Nusman et al., manuscript in preparation). A semi-quantitative scoring method like the one developed for MRI of the wrist in JIA patients may be an alternative approach worth investigating because it may overcome drawbacks of the measurement-based JAMRIS method (e.g. variety in pathology boundary at different locations or susceptibility to variation in the measurements due to the small dimensions of the synovium and an in-plane resolution of $0.39 \mathrm{~mm}^{2}$ ) [27].

The procedure of standardized assessment of JIA pathology on MRI of the knee compared with unaffected children as shown in the current study is analogous to the procedures in the JIA wrist. After development of a (preliminary) MRI scoring system for the wrist, data on the MRI appearance of the wrist in healthy children led to redefining of the scoring system for pathology [28-30]. Consequently, revised versions of the original scoring system were developed and validated in the wrist [27, 31, 32].

Our study has some limitations though. Besides the autoinflammatory status and delay in timing of contrast in our control cohort as discussed above, the discrepancy in field strength of the MRI between the JIA group (1.0 T) and control group $(1.5 \mathrm{~T})$ is another potential limitation. However, both readers did not experience any problems with the MRI quality nor were they able to identify JIA or unaffected children on the basis of image quality differences. Also, the lack of fluidsensitive MRI sequences and other directions (due to time restrictions in the unaffected children) hampers adequate interpretation of synovial fluid and thickening.

In conclusion, mild enhancing synovial thickening can be identified upon MRI of the knee in a cohort of children unaffected by clinical arthritis. Infrapatellar and cruciate ligament locations are unique sites affected by the inflammatory process of JIA when compared to the data in our unaffected children cohort. The existing JAMRIS system for the assessment of synovial inflammation in JIA needs further refinement to distinguish synovitis in JIA from enhancement in unaffected children. The introduction of location-weighting to JAMRIS to improve the score for synovial thickening needs further development and validation in a larger JIA sample of patients previously imaged and scored according to the non-revised JAMRIS methodology.

Acknowledgments The scientific guarantor of this publication is Mario Maas. The authors of this manuscript declare no relationships with any companies whose products or services may be related to the subject matter of the article. This study is supported by a grant from the Dutch Arthritis Association (grant number 2013_1_018). No complex statistical methods were necessary for this paper. Institutional review board approval by the Medical Ethics Committee at the Academic Medical Center in Amsterdam was obtained. Written informed consent was obtained from all subjects (parents and patients above 12 years of age) in this study. None of the study subjects have been previously reported. Methodology: prospective, observational, performed at one institution.

Open Access This article is distributed under the terms of the Creative Commons Attribution-NonCommercial 4.0 International License (http:// creativecommons.org/licenses/by-nc/4.0/), which permits any noncommercial use, distribution, and reproduction in any medium, provided you give appropriate credit to the original author(s) and the source, provide a link to the Creative Commons license, and indicate if changes were made.

\section{References}

1. Wallace CA, Giannini EH, Spalding SJ et al (2012) Trial of early aggressive therapy in polyarticular juvenile idiopathic arthritis. Arthritis Rheum 64:2012-2021

2. Wallace CA, Giannini EH, Spalding SJ et al (2014) Clinically inactive disease in a cohort of children with new-onset polyarticular juvenile idiopathic arthritis treated with early aggressive therapy: time to achievement, total duration, and predictors. J Rheumatol 41: $1163-1170$

3. Tynjälä P, Vähäsalo $P$, Tarkiainen $M$ et al (2011) Aggressive combination drug therapy in very early polyarticular juvenile idiopathic arthritis (ACUTE-JIA): a multicentre randomised open-label clinical trial. Ann Rheum Dis 70:1605-1612

4. Magni-Manzoni S, Malattia C, Lanni S, Ravelli A (2012) Advances and challenges in imaging in juvenile idiopathic arthritis. Nat Rev Rheumatol 8:329-336 
5. Hemke R, van Rossum MA, van Veenendaal M et al (2013) Reliability and responsiveness of the Juvenile Arthritis MRI Scoring (JAMRIS) system for the knee. Eur Radiol 23:1075-1083

6. Hemke R, Kuijpers TW, van den Berg JM et al (2013) The diagnostic accuracy of unenhanced MRI in the assessment of joint abnormalities in juvenile idiopathic arthritis. Eur Radiol 23:19982004

7. Stomp W, Krabben A, van der Heijde D et al (2015) Aiming for a simpler early arthritis MRI protocol: can Gd contrast administration be eliminated? Eur Radiol 25:1520-1527

8. Eshed I, Krabbe S, Ostergaard M et al (2015) Influence of field strength, coil type and image resolution on assessment of synovitis by unenhanced MRI-a comparison with contrast-enhanced MRI. Eur Radiol 25:1059-1067

9. Hemke R, van Veenendaal M, Kuijpers TW, van Rossum MA, Maas M (2012) Increasing feasibility and patient comfort of MRI in children with juvenile idiopathic arthritis. Pediatr Radiol 42:440448

10. Hemke R, Doria AS, Tzaribachev N, Maas M, van der Heijde DM, van Rossum MA (2014) Selecting magnetic resonance imaging (MRI) outcome measures for juvenile idiopathic arthritis (JIA) clinical trials: first report of the MRI in JIA special interest group. J Rheumatol 41:354-358

11. McQueen F, Østergaard M, Peterfy C et al (2005) Pitfalls in scoring MR images of rheumatoid arthritis wrist and metacarpophalangeal joints. Ann Rheum Dis 64:i48-i55

12. Partik B, Rand T, Pretterklieber ML, Voracek M, Hoermann M, Helbich TH (2002) Patterns of gadopentetate-enhanced MR imaging of radiocarpal joints of healthy subjects. AJR Am J Roentgenol 179:193-197

13. Boegård T, Johansson A, Rudling O, Petersson I, Forslind K, Jonsson K (1996) Gadolinium-DTPA-enhanced MR imaging in asymptomatic knees. Acta Radiol 37:877-882

14. Hyams JS, Ferry GD, Mandel FS et al (1991) Development and validation of a pediatric Crohn's disease activity index. J Pediatr Gastroenterol Nutr 12:439-447

15. Turner D, Otley AR, Mack D et al (2007) Development, validation, and evaluation of a pediatric ulcerative colitis activity index: a prospective multicenter study. Gastroenterology 133:423-432

16. Foster HE, Kay LJ, Friswell M, Coady D, Myers A (2006) Musculoskeletal screening examination (pGALS) for school-age children based on the adult GALS screen. Arthritis Rheum 55: 709-716

17. Consolaro A, Ruperto N, Bazso A et al (2009) Development and validation of a composite disease activity score for juvenile idiopathic arthritis. Arthritis Rheum 61:658-666

18. Nusman CM, Hemke R, Schonenberg D et al (2014) Distribution pattern of MRI abnormalities within the knee and wrist of juvenile idiopathic arthritis patients: signature of disease activity. AJR Am J Roentgenol 202:W439-W446

19. Østergaard M, Klarlund M (2001) Importance of timing of postcontrast MRI in rheumatoid arthritis: what happens during the first
60 minutes after IV gadolinium-DTPA? Ann Rheum Dis 60:10501054

20. Jibri Z, Martin D, Mansour R, Kamath S (2012) The association of infrapatellar fat pad oedema with patellar maltracking: a casecontrol study. Skelet Radiol 41:925-931

21. Østergaard M, Hansen M, Stoltenberg M, Lorenzen I (1996) Quantitative assessment of the synovial membrane in the rheumatoid wrist: an easily obtained MRI score reflects the synovial volume. Br J Rheumatol 35:965-971

22. Dotson JL, Hyams JS, Markowitz J et al (2010) Extraintestinal manifestations of pediatric inflammatory bowel disease and their relation to disease type and severity. J Pediatr Gastroenterol Nutr 51:140-145

23. Jose FA, Garnett EA, Vittinghoff E et al (2009) Development of extraintestinal manifestations in pediatric patients with inflammatory bowel disease. Inflamm Bowel Dis 15:63-68

24. Agarwal V, Kumar M, Singh JK, Rathore RK, Misra R, Gupta RK (2009) Diffusion tensor anisotropy magnetic resonance imaging: a new tool to assess synovial inflammation. Rheumatology (Oxford) 48:378-382

25. Hemke R, van Veenendaal M, van den Berg JM et al (2014) Oneyear followup study on clinical findings and changes in magnetic resonance imaging-based disease activity scores in juvenile idiopathic arthritis. J Rheumatol 41:119-127

26. Hemke R, Maas M, van Veenendaal M et al (2014) Contrastenhanced MRI compared with the physical examination in the evaluation of disease activity in juvenile idiopathic arthritis. Eur Radiol 24:327-334

27. Damasio MB, Malattia C, Tanturri de Horatio L et al (2012) MRI of the wrist in juvenile idiopathic arthritis: proposal of a paediatric synovitis score by a consensus of an international working group. Results of a multicentre reliability study. Pediatr Radiol 42:10471055

28. Malattia C, Damasio MB, Pistorio A et al (2011) Development and preliminary validation of a paediatric-targeted MRI scoring system for the assessment of disease activity and damage in juvenile idiopathic arthritis. Ann Rheum Dis 70:440-446

29. Müller LS, Avenarius D, Damasio B et al (2011) The paediatric wrist revisited: redefining MR findings in healthy children. Ann Rheum Dis 70:605-610

30. Boavida P, Hargunani R, Owens CM, Rosendahl K (2012) Magnetic resonance imaging and radiographic assessment of carpal depressions in children with juvenile idiopathic arthritis: normal variants or erosions? J Rheumatol 39:645-650

31. Lambot K, Boavida P, Damasio MB et al (2013) MRI assessment of tenosynovitis in children with juvenile idiopathic arthritis: inter- and intra-observer variability. Pediatr Radiol 43:796-802

32. Tanturri de Horatio L, Damasio B, Barbuti D et al (2012) MRI assessment of bone marrow in children with juvenile idiopathic arthritis: intra- and inter-observer variability. Pediatr Radiol 42: 714-720 